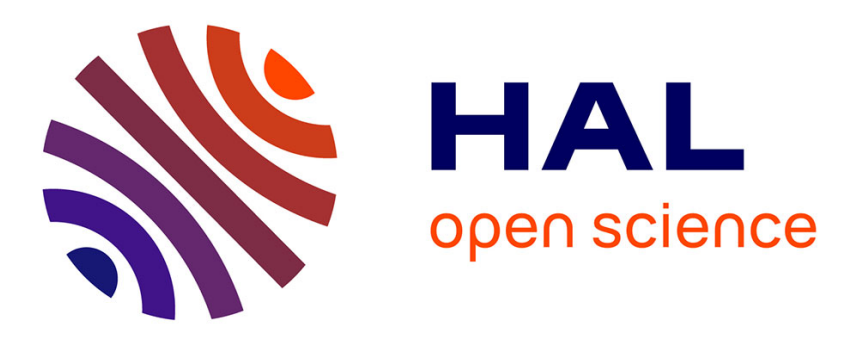

\title{
Ligne à Grande Vitesse et marchés immobiliers résidentiels à Reims : entre attractivité, aménités et anticipations
}

Sylvie Bazin, Christophe Beckerich, Marie Delaplace, Armand Colin

\section{To cite this version:}

Sylvie Bazin, Christophe Beckerich, Marie Delaplace, Armand Colin. Ligne à Grande Vitesse et marchés immobiliers résidentiels à Reims : entre attractivité, aménités et anticipations. Revue d'économie régionale et urbaine, 2010, mai (2), pp.3136336. 10.3917/reru.102.0313 . hal-02071503

\section{HAL Id: hal-02071503 \\ https://hal.univ-reims.fr/hal-02071503}

Submitted on 18 Mar 2019

HAL is a multi-disciplinary open access archive for the deposit and dissemination of scientific research documents, whether they are published or not. The documents may come from teaching and research institutions in France or abroad, or from public or private research centers.
L'archive ouverte pluridisciplinaire HAL, est destinée au dépôt et à la diffusion de documents scientifiques de niveau recherche, publiés ou non, émanant des établissements d'enseignement et de recherche français ou étrangers, des laboratoires publics ou privés. 


\section{LIGNE À GRANDE VITESSE ET MARCHÉS IMMOBILIERS RÉSIDENTIELS À REIMS : ENTRE ATTRACTIVITÉ, AMÉNITÉS ET ANTICIPATIONS}

Sylvie Bazin, Christophe Beckerich, Marie Delaplace

Armand Colin | « Revue d'Économie Régionale \& Urbaine »

2010/2 mai | pages 313 à 336

ISSN 0180-7307

ISBN 9782200926533

Article disponible en ligne à l'adresse :

http://www.cairn.info/revue-d-economie-regionale-et-urbaine-2010-2-page-313.htm

\section{!Pour citer cet article :}

Sylvie Bazin et al., «Ligne à Grande Vitesse et marchés immobiliers résidentiels à Reims : entre attractivité, aménités et anticipations », Revue d'Économie Régionale \& Urbaine 2010/2 (mai), p. 313-336.

DOI 10.3917/reru.102.0313

Distribution électronique Cairn.info pour Armand Colin.

(C) Armand Colin. Tous droits réservés pour tous pays.

La reproduction ou représentation de cet article, notamment par photocopie, n'est autorisée que dans les limites des conditions générales d'utilisation du site ou, le cas échéant, des conditions générales de la licence souscrite par votre établissement. Toute autre reproduction ou représentation, en tout ou partie, sous quelque forme et de quelque manière que ce soit, est interdite sauf accord préalable et écrit de l'éditeur, en dehors des cas prévus par la législation en vigueur en France. Il est précisé que son stockage dans une base de données est également interdit. 


\title{
Ligne à Grande Vitesse et marchés immobiliers résidentiels à Reims : entre attractivité, aménités et anticipations*
}

\section{High Speed Rail Link and housing markets in Rheims : between attractiveness, amenities and expectations}

\author{
Sylvie BAZIN \\ Christophe BECKERICH \\ Marie DeLAPLACE \\ Maîtres de conférences \\ Université de Reims Champagne-Ardenne \\ Laboratoire OMI \\ 57bis, rue Pierre Taittinger \\ 51096 Reims cedex \\ Secrétariat : 0326913801 \\ christophe.beckerich@univ-reims.fr**
}

Mots-clés : marché immobilier, Ligne à Grande Vitesse, anticipations.

Keywords : housing market, High Speed Rail Link, expectations.

Classification JEL : R21, R31, R40

\footnotetext{
*Première version reçue, juin 2008 ; version finale, décembre 2008.

***Auteur correspondant.
} 


\section{Résumé}

Depuis l'annonce de la création de la LGV Est-européenne en 2000, de nombreux acteurs considèrent que la croissance des prix de l'immobilier à Reims est imputable au TGV et à l'arrivée possible de nouveaux ménages et que cette tendance n'ira qu'en s'accentuant. A l'aide de la littérature économique relative au fonctionnement des marchés immobiliers et par l'analyse des transactions immobilières individuelles de 1999 à 2006 dans l'aire urbaine de Reims, nous montrons que ce constat doit être nuancé. Si le TGV apparaît comme un élément qui a participé à la croissance des prix de manière très localisée et à une redynamisation de la promotion immobilière, ce n'est pas en raison de l'arrivée massive de nouveaux ménages, mais d'anticipations par les acteurs.

\section{Summary}

Since the creation of the East European High Speed Rail Link (LGV) was announced in 2000, many observers have considered that housing price growth in Rheims could be directly attributed to the TGV (high-speed train) and to the possible influx of new households, and that these changes would inevitably become more pronounced. Drawing on the economic literature on property markets and the analysis of housing transactions in Rheims between 1999 and 2006, we show that this conclusion is not quite so clear cut. The TGV may appear to have been a contributory factor to price rises in a very localized way, and to have fueled property development but not due to any huge influx of new households. In fact, it results from the expectations of actors.

\section{- 1 - \\ Introduction}

Depuis le 10 juin 2007, la LGV Est-européenne dessert les principales agglomérations du Nord-Est de la France. Reims bénéficie de la desserte la plus importante en Champagne-Ardenne en termes de fréquence grâce à deux gares : la gare de Reims et la nouvelle gare TGV Champagne-Ardenne localisée à Bezannes ${ }^{1}$. La première accueille 8 allers et retours quotidiens qui permettent de rejoindre la gare de l'Est à Paris en 45 minutes contre 1 h 35 auparavant, soit une baisse du temps de parcours de $53 \%$. La seconde permet d'assurer la connexion de la région aux gares TGV d'Ile-de-France (Massy, Marne-la-Vallée et l'aéroport Roissy-Charles de Gaulle) et aux réseaux à grande vitesse du Nord, de l'Ouest et du Sud-ouest de la France sans transit par Paris (9 allers et retours dans la journée), ainsi que deux allers et retours sur Paris. Elle permet également de rejoindre (mais avec correspondance) des grandes villes européennes comme Londres, Bruxelles, Francfort, Bâle et Zurich ainsi que le réseau TGV Sud et Sud-Est ${ }^{2}$.

Cette desserte a suscité de fortes attentes de la part des acteurs économiques et politiques : dynamisation de l'économie, développement d'activités tertiaires et attraction de nouvelles entreprises et de ménages. Ces espoirs sont également accompagnés de craintes quant à la transformation de Reims en cité-dortoir et à l'augmentation des prix immobiliers qui serait associée à cette mise en service. Ainsi, une Ligne à Grande Vitesse (LGV) suscite encore de nombreux espoirs et craintes malgré de multiples réfutations académiques du concept des effets structurants (OFFNER, 1993). 
Cet article s'attache à analyser les conséquences de l'arrivée de la LGV Esteuropéenne, avant sa mise en service, sur l'évolution des marchés immobiliers résidentiels dans l'agglomération de Reims ${ }^{3}$ et ce jusqu'en 2006. L'objectif est d'identifier si certaines caractéristiques des marchés immobiliers à Reims ont connu une évolution qui serait liée à la perspective de la mise en service de la LGV Esteuropéenne.

Après avoir mis en évidence la façon dont une LGV peut influer sur les évolutions des marchés immobiliers résidentiels (section II), l'analyse des transactions immobilières individuelles dans l'agglomération de Reims de 1999 à $2006^{4}$ nous permettra de montrer que les évolutions enregistrées à Reims résultent à la fois des politiques publiques visant à changer l'image du quartier de la gare et des anticipations par les ménages et les investisseurs quant aux effets supposés de la mise en service de la LGV en termes d'attractivité (section III).

\section{- 2 - \\ Marchés immobiliers résidentiels et Ligne à Grande Vitesse}

Les marchés immobiliers locaux sont affectés par de nombreux facteurs. C'est le cas des facteurs macro-économiques qui participent à la formation des prix. Les marchés immobiliers locaux sont ainsi connectés à l'évolution nationale de l'immobilier ${ }^{5}$. Cette dernière est elle-même influencée, d'une part, par les variables déterminantes de l'économie (inflation, croissance, taux d'intérêt, etc.), d'autre part, par l'évolution des marchés des autres actifs et, enfin, par les politiques monétaires et fiscales qui conditionnent l'arbitrage avec d'autres placements. Les marchés immobiliers sont également liés aux évolutions démographiques et aux modifications des modes de vie (monoparentalité, divorce, etc.) qui influent sur le nombre de ménages et donc sur la demande de logements. Mais ils connaissent également des évolutions des prix résultant de déterminants locaux tels que la mise en service d'une desserte à grande vitesse. Cette dernière semble affecter les prix sur certains segments et ce de façon localisée (2.1.). Ces évolutions associées à l'arrivée d'une infrastructure de transport peuvent résulter de changements d'image et des aménités de certains quartiers (2.2.) mais également des anticipations plus générales des acteurs quant à l'évolution des marchés au moment de la mise en service de la LGV (2.3.).

\subsection{Un surcroit d'attractivité limité et une augmentation localisée des prix immobiliers résultant d'une demande ciblée}

Pour des villes relativement proches de Paris, la mise en service d'une LGV conduit à une diminution importante des temps de parcours vers la capitale et ouvre de nouvelles possibilités de localisation aux ménages envisageant de déménager. Cela peut favoriser la venue de ménages franciliens souhaitant bénéficier de prix 
immobiliers plus faibles qu'en Ile-de-France et d'une qualité de vie différente. Inversement, une nouvelle desserte peut permettre à des ménages habitant dans les villes de province desservies de bénéficier du marché du travail francilien, tout en y conservant leur lieu de résidence. Ainsi, la LGV peut conduire à une augmentation de la demande de logements ${ }^{6}$.

Cependant, l'analyse des expériences passées relatives à la mise en place de dessertes ferroviaires à grande vitesse dans des agglomérations françaises proches de Paris montre le caractère marginal à court et moyen terme de ces implantations de ménages. Le TGV n'a jamais transformé ces agglomérations en cités dortoirs en raison d'une installation massive de nouveaux habitants (IAURIF, 2003). Toutefois, des ménages nouvellement installés dans les agglomérations desservies déclarent avoir choisi leur lieu de résidence du fait de l'existence d'une liaison à grande vitesse avec Paris (BAZIN et al., 2006a). Si leur poids demeure marginal, il peut entraîner un déséquilibre entre l'offre et la demande notamment sur certains segments étroits des marchés immobiliers. En effet, ces ménages, qui relèvent souvent de PCS élevées, ont des attentes spécifiques en matière de logement: maison individuelle avec jardin en centre-ville ou à proximité de la ville, ou grand appartement de qualité en centre-ville. Ces deux segments qui représentent des marchés limités dans la plupart des agglomérations françaises, sont alors caractérisés par une forte hausse des prix relativement aux autres types de biens lors de l'arrivée du TGV. Sur le long terme, cependant, les prix immobiliers moyens ne paraissent pas être plus élevés dans les villes $\mathrm{TGV}^{7}$.

La desserte TGV s'accompagne également de politiques publiques visant à améliorer l'image du ou des quartiers proches de la gare et qui modifient leurs aménités.

\subsection{Une capitalisation immobilière des aménités et de l'image de la ville résultant des politiques de valorisation du TGV}

Les transports participent à la formation des prix immobiliers comme le montrent les modèles de localisation des ménages (ALONSO, 1964 ; WINGO, 1961 ; MUTH, 1969 ; FUJITA, 1989) et les tests empiriques afférents (BAJIC, 1983 ; BECKERICH, 2001 ; RICS, 2002 ; Yiu et WONG, 2005 ; ArMSTRONG et RodigueZ, 2006). Mais une LGV, en modifiant les conditions d'accessibilité aux agglomérations desservies, ne peut être comparée à un réseau de transport collectif urbain ou à une nouvelle desserte routière ou autoroutière. En effet, le TGV est une infrastructure qui a pour vocation de relier les grands pôles urbains métropolitains entre eux et aux grandes infrastructures aéroportuaires $^{8}$. Aussi l'évolution de l'accessibilité n'est pas comparable à celle provoquée, par exemple, par la mise en place d'une ligne de tramway.

Si l'accessibilité modifiée par le TGV joue un rôle "secondaire » dans les choix résidentiels, l'arrivée de cette nouvelle infrastructure dans une agglomération conduit, cependant, les acteurs publics à mener des stratégies en vue de capter et d'accroître les bénéfices anticipés du changement d'image entraîné par la desserte TGV (BAZIN et al., 2006b). Les collectivités locales élaborent des diagnostics territoriaux à cette occasion 
qui intègrent la nouvelle donne ferroviaire. Elles accompagnent le changement d'image par la volonté de restructurer la ville. Les premières réalisations concernent les quartiers autour de la gare. Ces derniers (et plus particulièrement les quartiers d'arrière-gare) se transforment souvent en « quartiers d'affaires " avec une complète révision de leurs fonctions et donc de leur urbanisme. Les centres-villes sont souvent rénovés, les réseaux de transport urbain restructurés afin d'améliorer la desserte de l'agglomération à partir de la gare ${ }^{9}$.

Ainsi au-delà des opérations affectant directement l'immobilier résidentiel, une modification plus globale de l'image de la ville peut se produire. Dans la perspective de l'accueil d'entreprises nouvelles, des zones d'activités sont créées. Généralement, les promoteurs lancent des opérations immobilières souvent haut de gamme. Les tourismes d'affaires et urbain sont également affectés puisque le TGV peut, dans certains cas, dynamiser ces activités (BAZIN et al., à paraître). La destination peut faire l'objet d'une mise en tourisme, ce qui peut se traduire par une restructuration de l'offre hôtelière, par la mise en valeur du patrimoine architectural et culturel, etc.

Ces évolutions conduisent à transformer les aménités de certains quartiers de l'agglomération et l'image de la ville pour les acteurs économiques extérieurs mais également pour les acteurs locaux et plus particulièrement pour les ménages. Cela peut modifier leurs choix résidentiels et participer à l'évolution différenciée des prix immobiliers intra-urbains. En effet, ces derniers en achetant un logement procèdent à l'achat d'un panier de caractéristiques spécifiant le logement. Ce dernier se définit alors par ses qualités intrinsèques (superficie, nombre de pièces, nombre de salles de bain, exposition, qualité de la construction, etc.) et par les attributs de lieux (PAPAgEOrgiou, 1973, 1990 ; FuJITA, 1989 ; BOWES et IHLANFELDT, 2001). Ces attributs de lieux regroupent les caractéristiques de la localisation du logement (proximité des zones d'emploi, des commerces et des services publics, qualité de l'environnement naturel et social). Ces éléments agissent directement sur la formation des prix immobiliers par l'intermédiaire de leurs externalités (FUJTA, 1989).

Ainsi suite à une modification des aménagements publics et à la transformation du bâti résultant de stratégies d'acteurs pour capter d'éventuels effets bénéfiques de la LGV, des quartiers peu prisés peuvent devenir recherchés et caractérisés par d'importantes évolutions des prix immobiliers. Toutefois, les évolutions observées à l'approche de la mise en service de la desserte TGV ne peuvent être expliquées uniquement par ces éléments.

\subsection{LGV et anticipations sur les marchés immobiliers}

La mise en service d'une LGV se traduit par des anticipations qui, pour certaines, reposent sur des croyances sociales ${ }^{10}$. Ainsi les acteurs économiques sur les territoires desservis sont généralement convaincus d'une forte progression globale des prix en raison d'une arrivée massive de population, qui ne s'est pourtant jamais réellement déroulée dans les expériences passées (cf. 2.1.). 
Ces croyances sociales sont diffusées par la presse et les professionnels. Ces derniers ont intérêt à les entretenir puisque leurs revenus sont proportionnels à la valeur des biens immobiliers. Par ailleurs, l'indigence de l'information statistique sur les prix immobiliers et fonciers, en France, malgré les efforts des Chambres des notaires, conduit les ménages à penser que les augmentations des prix dans certains quartiers proches de la gare ou sur certains segments concernent l'ensemble du marché. Ceci est accentué par les propos tenus dans la presse qui mettent en exergue des cas singuliers de progression des prix souvent « à dire d'experts » et qui s'avèrent généralement peu représentatifs.

Ces croyances sociales vont participer à la structuration des anticipations des acteurs.

Les ménages sont ainsi susceptibles d'anticiper une augmentation des prix immobiliers à l'approche de la mise en service de la LGV. Ils peuvent accepter de payer leur logement au-dessus de son prix "réel " puisqu'ils anticipent que les prix immobiliers vont continuer à progresser. En outre, certains d'entre eux peuvent être tentés de précipiter leur achat de peur que les prix immobiliers les empêchent d'acheter à l'avenir (RENARD, 2003). Enfin, ils peuvent anticiper les évolutions futures du niveau d'accessibilité et des aménités dans l'agglomération et le quartier (MCDONALD et OsujI, 1995 ; CHAU et NG, 1998; DEYMIER, 2005), en raison des politiques publiques initiées effectivement par les collectivités (cf. 2.2).

Les investisseurs locaux, nationaux et internationaux peuvent également anticiper le fait que les prix immobiliers seront en forte progression au moment de la mise en service de la LGV. Ils peuvent acheter certains biens en espérant réaliser une plus-value après cette mise en service ou réaliser des opérations de rénovation ou de réhabilitation de logements à proximité de la gare pour bénéficier des changements d'aménités du quartier.

Les promoteurs peuvent aussi anticiper une hausse des prix leur permettant de rendre viables des projets immobiliers. En effet, l'analyse des expériences passées met en lumière une certaine redynamisation de la construction immobilière dans les agglomérations desservies aussi bien sur le segment de l'offre immobilière de bureaux et de locaux d'entreprises ${ }^{11}$ que sur l'immobilier résidentiel ${ }^{12}$. L'anticipation d'une hausse des prix favorise la réalisation de nouveaux programmes immobiliers puisqu'elle permet aux promoteurs de proposer un prix plus élevé aux propriétaires des emplacements qu'ils convoitent, les incitant ainsi à vendre ${ }^{13}$. Cette offre immobilière supplémentaire est souvent disponible au moment de la mise en service de la desserte à grande vitesse et conduit à légitimer l'idée d'une progression des prix dans les villes nouvellement desservies par une LGV puisque les prix fixés par les promoteurs apparaissent comme les prix du marché.

L'ensemble des anticipations des acteurs aussi bien du côté de l'offre que de la demande concourt ainsi à favoriser l'augmentation des prix en amont de la mise en service de la LGV. Certaines anticipations sont avérées. Elles reposent sur des éléments tels que les aménités nouvelles résultant du changement d'image et peuvent 
être qualifiées de rationnelles (CORNUEL, 1999). D'autres, comme l'afflux massif de ménages sont infondées et peuvent être qualifiées de naïves (NAPPI-CHOULET, 1994).

\section{- 3 - \\ La LGV Est-européenne et les marchés immobiliers résidentiels dans l'agglomération de Reims de 1999 à 2006}

L'annonce officielle de la réalisation de la Ligne à Grande Vitesse Est-européenne en 2000 a été faite alors que les marchés immobiliers dans l'aire urbaine de Reims, souvent considérés comme singuliers, connaissaient une phase d'apathie. Depuis cette période, ces marchés ont connu le retour d'un certain dynamisme et une forte augmentation des prix quel que soit le segment (3.1). Les déterminants de ces évolutions de prix peuvent d'abord résulter de tensions liées aux évolutions démographiques propres à l'agglomération de Reims en lien ou pas avec le TGV et/ou de l'arrivée d'investisseurs parisiens (3.2). Toutefois, l'augmentation globale des prix semble être peu liée au TGV, même si certaines anticipations parfois spéculatives, et qui traduisent l'existence des croyances sociales, sont perceptibles (3.3).

\subsection{Une relative croissance des prix immobiliers et un dynamisme des marchés retrouvé}

Le marché rémois est considéré comme atypique par rapport aux grandes agglomérations françaises. En effet, Reims présente la spécificité de détenir le record national des agglomérations de plus de 150000 habitants en termes de pourcentage de logements collectifs HLM. Elle conjugue à la fois le plus fort taux de locataires (63,50\% des résidences principales) et la part la plus importante de locataires HLM (40,20\% des résidences principales). Du fait de l'importance du patrimoine immobilier géré par les organismes bailleurs, le pourcentage de propriétaires y est alors plus restreint qu'ailleurs. Ainsi, l'agglomération rémoise occupe la dernière place en ce qui concerne la part des propriétaires occupants dans les agglomérations de plus de 150000 habitants $^{14}$.

Depuis 1999, ce marché atypique a connu, toutefois, une progression des prix immobiliers moindre que sur d'autres territoires (3.1.1) ainsi qu'une croissance de la construction de logements neufs (3.1.2).

\subsubsection{Une progression contenue des prix immobiliers}

À l'instar du marché immobilier français ${ }^{15}$, et quel que soit le segment, les prix immobiliers dans l'agglomération de Reims ont fortement progressé. Ainsi, entre 1999 et 2006, le montant moyen des transactions immobilières a progressé respectivement de 93,7 \% et de 87,5\% pour les maisons anciennes et neuves (cf. tableau 1). 


\section{Tableau 1 - Évolution du montant moyen des transactions immobilières selon le segment du marché de 1999 à 2006 - Base 100 en 1999}

\begin{tabular}{|c|c|c|}
\hline Année & $\begin{array}{c}\text { Maisons } \\
\text { anciennes }\end{array}$ & $\begin{array}{c}\text { Maisons } \\
\text { neuves }\end{array}$ \\
\hline 1999 & 100,0 & 100,0 \\
\hline 2000 & 111,1 & 122,4 \\
\hline 2001 & 119,0 & 126,3 \\
\hline 2002 & 123,5 & 108,7 \\
\hline 2003 & 130,7 & 127,6 \\
\hline 2004 & 148,2 & 154,1 \\
\hline 2005 & 167,5 & 158,6 \\
\hline 2006 & 193,7 & 187,5 \\
\hline
\end{tabular}

Source : Les auteurs à partir des données PERVAL

Cette croissance est également observable pour le prix moyen au mètre carré des appartements anciens et neufs qui progresse respectivement de 87,8 \% et de 52,4\% entre 1999 et 2006 (cf. tableau 2). L'augmentation des prix sur tous les segments tend à s'amplifier en 2004 et 2005. Par exemple, pour les appartements anciens, le prix au $\mathrm{m}^{2}$ évolue à un rythme à un chiffre jusqu'en 2003 (7,6\% en 2003), puis à deux chiffres en 2004 et 2005 (respectivement $+17,4 \%$ et $+16,3 \%)^{16}$. En 2006, la progression est plus mesurée et de l'ordre de 9,7\%. Pour les appartements neufs, les prix connaissent une très forte progression en $2005(+37,1 \%)$ suivie d'une légère décélération en 2006.

Tableau 2 - Évolution du prix moyen au $\mathrm{m}^{2}$ selon le segment du marché de 1999 à 2006 - Base 100 en 1999

\begin{tabular}{|c|c|c|}
\hline Année & $\begin{array}{c}\text { Appartements } \\
\text { anciens }\end{array}$ & $\begin{array}{c}\text { Appartements } \\
\text { neufs }\end{array}$ \\
\hline 1999 & 100,0 & 100,0 \\
\hline 2000 & 108,4 & 103,3 \\
\hline 2001 & 109,5 & 101,8 \\
\hline 2002 & 116,8 & 102,3 \\
\hline 2003 & 125,7 & 112,1 \\
\hline 2004 & 147,6 & 114,0 \\
\hline 2005 & 171,6 & 156,3 \\
\hline 2006 & 187,8 & 152,4 \\
\hline
\end{tabular}

Source : Les auteurs à partir des données PERVAL

Toutefois, les rythmes de croissance observés dans l'agglomération rémoise entre 1999 et 2006 sont inférieurs à ceux observés en France. Le prix moyen par m² des appartements anciens dans les agglomérations de plus de 10000 habitants de province est passé de l'indice 89,7 à l'indice 213,1 entre le premier trimestre 1999 et le dernier trimestre 2006, soit une progression de 137 \% (cf. figure 1). Pour la même période, pour les maisons anciennes en province, l'augmentation est de $115 \%$ 
avec un indice passant de 87,4 à 188,4 (INSEE, Chambre Supérieure du Notariat, 2007). Une accélération de la hausse est observée sur le marché local à partir de 2004 comme au niveau national. Cependant, malgré des différences de méthode de calcul de ces indices et des chiffres présentés pour l'agglomération de Reims, la progression des prix a été plus faible que celle observée pour la France de province. Cela pourrait s'expliquer par les effets d'une démographie contrastée (cf. 3.2.1) ou encore par un niveau de prix initialement plus élevé. Cette évolution se retrouve dans le classement des villes de plus de 150000 habitants selon le prix par $\mathrm{m}^{2}$ des appartements anciens. Reims était la 4eme ville la plus chère de France après Nice, Strasbourg et Lyon en 1999, la treizième ville la plus chère en 2003, et la dixième ville la plus chère en 2004 (Chambre des Notaires de la Marne, 2004) ${ }^{17}$.

\section{Figure 1 - Evolution comparée des prix des appartements anciens de 1999 à 2006 dans les agglomérations de plus de 10000 habitants et à Reims ${ }^{\mathrm{a}}$}

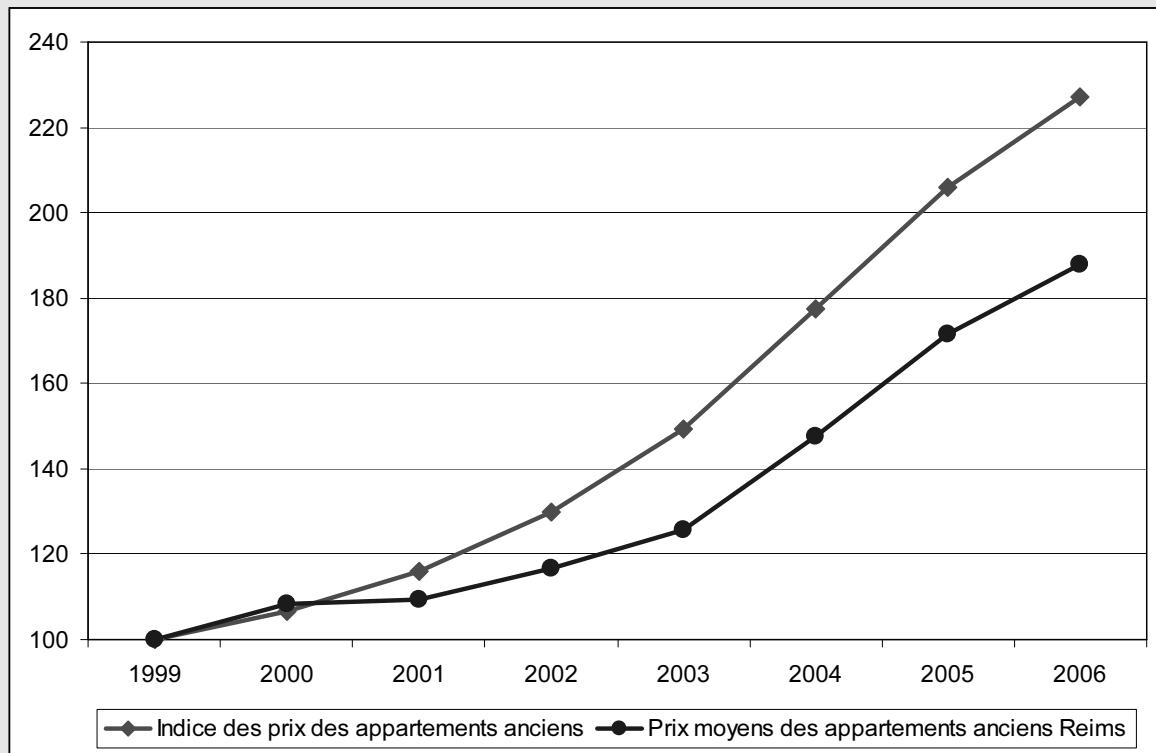

a. Les deux indicateurs ne sont pas calculés de la même façon. Dans le cas rémois, il s'agit d'un prix moyen et non pas un indice des prix comme calculé par I'INSEE.

\subsubsection{Le marché immobilier rémois en croissance après une phase d'apathie}

L'augmentation des prix s'est accompagnée d'une augmentation du nombre de transactions conformément à ce qui est observé, généralement, lors des phases de progression des prix immobiliers (cf. tableau 3$)^{18}$. Ainsi, le nombre de transactions se redresse sensiblement sur le segment des appartements neufs, après une phase d'apathie mais demeure en dessous du niveau de la fin des années 1990. 


\section{Tableau 3 - Évolution du nombre de transactions selon le segment du marché de 1999 à 2006 - Base 100 en 1999}

\begin{tabular}{|l|c|c|c|c|}
\hline Année & $\begin{array}{c}\text { Appartement } \\
\text { ancien }\end{array}$ & $\begin{array}{c}\text { Appartement } \\
\text { neuf }\end{array}$ & $\begin{array}{c}\text { Maison } \\
\text { ancienne }\end{array}$ & Maison neuve \\
\hline 1999 & 100,0 & 100,0 & 100,0 & 100,0 \\
\hline 2000 & 92,7 & 27,4 & 96,5 & 161,3 \\
\hline 2001 & 101,9 & 24,3 & 95,1 & 106,5 \\
\hline 2002 & 100,5 & 34,1 & 100,3 & 77,4 \\
\hline 2003 & 122,2 & 36,7 & 105,7 & 151,6 \\
\hline 2004 & 123,5 & 69,0 & 109,0 & 154,8 \\
\hline 2005 & 132,3 & 58,4 & 103,8 & 180,6 \\
\hline 2006 & 124,0 & 41,6 & 105,8 & 171,0 \\
\hline
\end{tabular}

Source : Les auteurs à partir des données PERVAL

Cette situation est en partie atypique par rapport à celle observée au niveau national pendant cette période. La croissance de l'immobilier, en France comme à Reims de 1997 à 2003, a été portée principalement par les maisons individuelles neuves localisées dans les communes rurales en périphérie des agglomérations urbaines. Ainsi, dans l'aire urbaine de Reims, le nombre de transactions progresse très fortement sur le marché des maisons neuves, à savoir $+71 \%$ entre 1999 et 2006. En revanche, entre 1997 et 2000, alors que la construction de logements collectifs a été stimulée par les programmes dits "PÉRISSOL » (MTETM/SESP, 2006) et que jusqu'en 2003, le marché a connu une phase de consolidation dans les différents segments, dans le cas rémois, cela s'est transformé en un quasi-arrêt de la construction. Seulement 600 logements ont été construits en 2003 contre 1000 par an dans la décennie précédente. Sur le territoire de la commune de Reims, la production de logements s'est effondrée en diminuant des deux tiers durant la période ${ }^{19}$.

L'absence de foncier disponible à Reims semble, en partie, expliquer cette situation ${ }^{20}$. Par ailleurs, cette phase de fort déclin de la construction est concomitante à une phase d'incertitude par rapport au projet de la LGV Est-européenne. En effet, si le projet a été avalisé en 2000, après une longue phase de négociation entre les acteurs, les travaux n'ont réellement débuté qu'en 2002. Par ailleurs, l'annonce en 2002 du report à juin 2007 de la mise en service de la LGV prévue initialement en 2006 a contribué à rendre les opérateurs immobiliers prudents et attentistes. L'analyse du jeu des acteurs économiques et politiques montre que la prise de conscience de l'arrivée du TGV ne s'est réalisée qu'à partir du moment où les travaux de génie civil du projet ont été visibles aux portes de Reims, à savoir à partir de l'année 2004.

Depuis 2004 au niveau national, et de façon plus marquée depuis 2005 pour Reims, le marché est à nouveau en phase de croissance notamment dans le segment du logement collectif soutenu par le dispositif fiscal «DE ROBIEN » d'incitation à l'investissement locatif et le volet logement du plan «BORLOO » de cohésion sociale (INSEE Champagne-Ardenne, 2006). Sur la ville de Reims, de nombreux projets sont ainsi en cours de réalisation ${ }^{21}$. 


\subsection{L'évolution des prix immobiliers résidentiels : demande locale versus migration résidentielle?}

L'augmentation des prix et le développement de l'activité de construction peuvent découler d'une progression de la demande résultant d'un dynamisme démographique endogène (3.2.1) ou d'une attractivité renouvelée de l'agglomération rémoise pour les ménages franciliens en raison de l'arrivée imminente de la LGV (3.2.2).

\subsubsection{Le maintien d'une demande forte malgré des évolutions démographiques contrastées}

La population de l'aire urbaine de Reims n'est pas caractérisée par un dynamisme marqué. Ainsi, si elle n'a cessé de croître depuis 1975, sa croissance ralentit sur la dernière période intercensitaire ${ }^{22}$, à la fois en raison d'une détérioration du solde migratoire mais également en raison d'un ralentissement de la croissance due au solde naturel. Depuis 1999, l'évolution semble encore plus défavorable. Ainsi, le territoire correspondant au $\mathrm{SCOT}^{23}$ de la région rémoise - qui est un peu plus restreint que l'aire urbaine de Reims - semble connaître un essoufflement démographique (INSEE, 2006). Il en va de même pour la ville de Reims.

Selon les dernières enquêtes annuelles de recensement, le nombre d'habitants rémois n'était plus que de 184000 au 1er janvier 2007 contre 187206 en 1999. De nombreux ménages ont choisi de s'installer dans le périurbain ou le rural proche. Ainsi entre 1999 et 2005, 1781 ménages habitant le territoire de la communauté d'agglomération " Reims Métropole » ont obtenu une autorisation de construire une maison individuelle hors de l'agglomération rémoise, soit 52 \% de plus qu'entre 1992 et 1998. Plus du tiers de ces autorisations porte sur une commune située au-delà du périmètre du SCOT (INSEE, 2006).

Toutefois, le nombre de ménages rémois est passé de 83262 en 1999 à 87757 en 2005 (INSEE, 2007) soit une progression de 5,4\%. La demande de logement ne semble pas avoir faibli parce que la baisse de la population a été compensée par l'augmentation du nombre de ménages en raison, notamment, des modifications des modes de vie (développement de la monoparentalité et des mono-ménages, vieillissement de la population, etc.).

Aussi, la progression des prix est-elle expliquée, en partie, par le maintien d'une demande de logements résultant de la croissance du nombre de ménages, augmentation qui n'est pas spécifique à l'agglomération rémoise ${ }^{24}$. Sur Reims, cependant, la croissance est moins forte que celle enregistrée pour la France de province. En effet, sur cette même période et pour la France de province, le nombre de ménages croît de 9,3 \% passant de 19299792 en 1999 à 21086261 en 2005 (INSEE, 2008).

\subsubsection{Une présence plus importante des acquéreurs franciliens qui n'explique pas à elle seule la progression des prix}

Le dynamisme de la demande peut également résulter d'une attractivité accrue de l'agglomération du fait de la desserte TGV qui pourrait se traduire par l'arrivée 
de franciliens et/ou d'investisseurs franciliens. L'analyse de l'origine des acheteurs permet d'éclairer ce point.

Les transactions sur les marchés immobiliers dans l'aire urbaine de Reims sont réalisées principalement par des acheteurs domiciliés dans le département de la Marne et, plus largement, en Champagne-Ardenne. Cependant, leur origine géographique tend à évoluer. Les acquéreurs localisés en Ile-de-France au moment de la vente sont plus nombreux en 2006 qu'en 1999 et leur part est toujours croissante dans les transactions (excepté pour les maisons neuves) avec une accélération en fin de période (cf. tableau 4). Sur le marché des appartements anciens en 2006, les franciliens représentent 5,5\% des acquéreurs alors qu'ils n'en représentaient que $1,6 \%$ en 1999. Sur le segment des maisons anciennes, le pourcentage des transactions effectuées par un acheteur francilien passe de 0,9\% à 2,5\%. Sur le segment des appartements neufs, la présence des franciliens est encore plus notable en fin de période. En 2006, 16,5\% des transactions concernant un appartement neuf ont pour acheteurs des franciliens contre 1,9\% en 1999. Toutefois, ces chiffres doivent être relativisés puisque ces situations ne concernent souvent que quelques dizaines de transactions. En outre, leur présence peut être sous estimée. En effet, il est rare que de nouveaux arrivants décident d'acheter immédiatement un bien immobilier, et souvent une phase de location précède l'acquisition.

\section{Tableau 4 - Évolution de la présence des acquéreurs franciliens sur les marchés immobiliers dans l'agglomération de Reims de 1999 à 2006}

\begin{tabular}{|c|c|c|c|c|c|c|c|c|c|c|c|c|}
\hline \multirow[b]{2}{*}{ Année } & \multicolumn{3}{|c|}{ Maisons anciennes } & \multicolumn{3}{|c|}{$\begin{array}{l}\text { Appartements } \\
\text { anciens }\end{array}$} & \multicolumn{3}{|c|}{ Maisons neuves } & \multicolumn{3}{|c|}{ Appartements neufs } \\
\hline & $\frac{n}{\frac{n}{\pi}}$ & 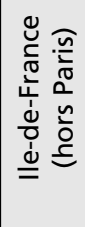 & 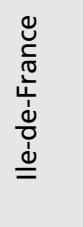 & $\frac{n}{\frac{\pi}{0}}$ & 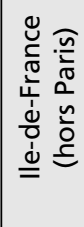 & 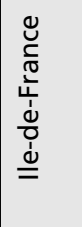 & $\frac{n}{\frac{\pi}{\pi}}$ & 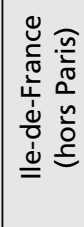 & 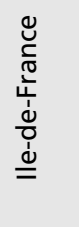 & $\frac{\pi}{\pi}$ & 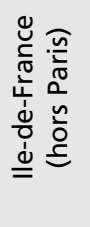 & 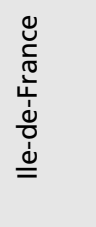 \\
\hline 1999 & $0,2 \%$ & $0,6 \%$ & $0,9 \%$ & $0,2 \%$ & $1,4 \%$ & $1,6 \%$ & $4,8 \%$ & $4,8 \%$ & $9,5 \%$ & $0,5 \%$ & $1,4 \%$ & $1,9 \%$ \\
\hline 2000 & $0,0 \%$ & $1,3 \%$ & $1,3 \%$ & $0,8 \%$ & $1,9 \%$ & $2,7 \%$ & $0,0 \%$ & $0,0 \%$ & $0,0 \%$ & $0,0 \%$ & $3,6 \%$ & $3,6 \%$ \\
\hline 2001 & $0,2 \%$ & $2,1 \%$ & $2,3 \%$ & $1,0 \%$ & $0,4 \%$ & $1,4 \%$ & $4,3 \%$ & $0,0 \%$ & $4,3 \%$ & $0,0 \%$ & $2,2 \%$ & $2,2 \%$ \\
\hline 2002 & $0,2 \%$ & $1,4 \%$ & $1,6 \%$ & $0,4 \%$ & $1,5 \%$ & $1,9 \%$ & $0,0 \%$ & $0,0 \%$ & $0,0 \%$ & $0,0 \%$ & $2,7 \%$ & $2,7 \%$ \\
\hline 200 & $0,4 \%$ & $0,8 \%$ & $1,2 \%$ & $1,3 \%$ & $1,9 \%$ & $3,2 \%$ & $0,0 \%$ & $0,0 \%$ & $0,0 \%$ & $0,0 \%$ & $1,6 \%$ & $1,6 \%$ \\
\hline 2004 & $0,4 \%$ & $1,7 \%$ & $2,2 \%$ & $2,1 \%$ & $2,6 \%$ & $4,7 \%$ & $0,0 \%$ & $0,0 \%$ & $0,0 \%$ & $2,1 \%$ & $8,6 \%$ & $10,7 \%$ \\
\hline 2005 & $0,6 \%$ & $1,3 \%$ & $1,9 \%$ & $2,0 \%$ & $3,0 \%$ & $5,0 \%$ & $3,2 \%$ & $0,0 \%$ & $3,2 \%$ & $1,6 \%$ & $4,9 \%$ & $6,5 \%$ \\
\hline 2006 & $0,6 \%$ & $1,9 \%$ & $2,5 \%$ & $1,8 \%$ & $3,7 \%$ & $5,5 \%$ & $0,0 \%$ & $0,0 \%$ & $0,0 \%$ & $3,3 \%$ & $13,2 \%$ & $16,5 \%$ \\
\hline
\end{tabular}

Source : Les auteurs à partir des données PERVAL

Plusieurs raisons peuvent être évoquées pour tenter d'expliquer cette prévalence des franciliens sur ces marchés.

Des ménages ont pu choisir de résider dans un espace où les prix immobiliers permettent encore d'accéder à la propriété tout en conservant leurs emplois en 
Ile-de-France. En effet, la progression des prix immobiliers en Ile-de-France depuis 1999 peut empêcher certains ménages d'accéder à la propriété : depuis 1999, les prix immobiliers à Paris ont doublé sur tous les segments et tous les départements franciliens à l'exception des Yvelines (Chambre des Notaires Paris Ile-de France, 2007). Ainsi, les prix moins élevés pratiqués dans l'aire urbaine de Reims par rapport à Paris et à de nombreuses communes d'Ile-de-France pourraient expliquer le choix de l'agglomération rémoise $e^{25}$.

Cependant, les coûts de transport réduisent en partie ou annulent l'intérêt financier d'une telle installation dans le cas d'un maintien du lieu de travail à Paris. En effet, le tarif classique de l'abonnement mensuel étant de 450 euros hors frais de réservation, le différentiel n'est plus aussi significatif pour des logements situés en couronne parisienne (BAZIN et al., 2006a) ${ }^{26}$.

Comme nous l'avons souligné (cf. 2.1), l'analyse des expériences passées montre que la mise en place d'une ligne à grande vitesse ne conduit pas à l'installation de nombreux ménages franciliens dans les villes nouvellement desservies (IAURIF, 2003). En revanche, des anticipations autour de l'arrivée du TGV ont pu conduire des investisseurs franciliens à s'intéresser au marché immobilier de l'agglomération rémoise et expliquer la présence plus importante des franciliens sur le marché de l'immobilier neuf.

\subsection{Anticipations liées au TGV et évolution de certaines caractéristiques des marchés immobiliers}

L'analyse des transactions immobilières dans l'agglomération rémoise conduit à penser que le TGV a été intégré ex ante dans les choix des acteurs et que cela se traduit, d'une part, dans les prix observés à proximité de la gare (3.3.1) et, d'autre part, par une accélération du taux de rotation de certains biens et par l'augmentation des transactions concernant les biens loués (3.3.2). Par ailleurs, de nouveaux acteurs qui anticipent une forte augmentation des prix au moment de la mise en service de la LGV Est-européenne apparaissent sur le marché (3.3.3).

\subsubsection{Des évolutions contrastées selon les segments et la localisation des biens}

Les prix des appartements anciens à Reims ont progressé en moyenne de 87,3\% de 1999 à 2006. Cependant, l'évolution globale des prix à l'échelle de Reims ne donne qu'une vision partielle de la situation puisque la progression des prix dépend de la localisation exacte du bien immobilier au sein de l'agglomération, les prix capitalisant les caractéristiques de l'environnement des biens immobiliers (cf. 2.2). Ceci est attesté par le test ANOVA qui réfute l'hypothèse d'égalité des moyennes des prix immobiliers au $\mathrm{m}^{2}$ entre quartiers ${ }^{27}$.

Ainsi, la progression varie de 39,2 \% à $115 \%$ selon le quartier. Les quartiers proches de la gare de Reims connaissent une progression supérieure à la moyenne. 
Dans les quartiers Clairmarais et Faubourg de Laon, les prix ont augmenté respectivement de $94,1 \%$ et de $101,7 \%$ (cf. tableau 5 et figure 2). La progression est très forte pour ces quartiers en 2004 et 2005. Toutefois, certains quartiers moins directement concernés par le TGV connaissent les mêmes évolutions.

\section{Tableau 5 - Évolution des prix des appartements anciens de 1999 à 2006 dans les quartiers de Reims (base 100 en 1999)}

\begin{tabular}{|l|c|c|c|c|c|c|c|c|}
\hline Quartier & 1999 & 2000 & 2001 & 2002 & 2003 & 2004 & 2005 & 2006 \\
\hline Centre & 100 & 108,7 & 107,5 & 118,6 & 130,0 & 145,9 & 172,1 & 196,4 \\
\hline Cernay & 100 & 95,7 & 98,1 & 101,8 & 113,0 & 131,0 & 156,5 & 155,4 \\
\hline Clairmarais & 100 & 100,2 & 111,4 & 118,5 & 121,8 & 144,6 & 181,5 & 194,1 \\
\hline Courlancy & 100 & 100,4 & 105,8 & 109,8 & 112,4 & 136,8 & 162,6 & 178,7 \\
\hline Croix-Rouge & 100 & 125,5 & 123,3 & 120,9 & 128,5 & 141,6 & 180,0 & 139,2 \\
\hline Jean-Jaurès & 100 & 103,9 & 116,6 & 127,7 & 133,6 & 164,1 & 196,3 & 215,0 \\
\hline La Neuvillette & 100 & 96,7 & 105,8 & 97,2 & 95,5 & 130,7 & 160,2 & 170,3 \\
\hline Laon & 100 & 107,4 & 109,7 & 114,7 & 126,4 & 152,8 & 178,8 & 201,7 \\
\hline Moissons & 100 & 105,0 & 104,3 & 115,1 & 122,2 & 149,1 & 173,3 & 185,0 \\
\hline Nord-Est & 100 & 114,8 & 113,6 & 131,3 & 154,2 & 161,3 & 162,2 & 176,3 \\
\hline Saint-André & 100 & 119,1 & 120,1 & 124,8 & 139,3 & 152,2 & 184,8 & 209,7 \\
\hline Saint-Anne & 100 & 105,0 & 102,0 & 113,5 & 135,9 & 155,0 & 187,1 & 213,5 \\
\hline Saint-Rémi & 100 & 102,8 & 113,7 & 120,2 & 129,4 & 157,9 & 168,6 & 177,5 \\
\hline Total & 100 & 108,0 & 109,3 & 116,4 & 125,6 & 147,3 & 173,3 & 187,3 \\
\hline
\end{tabular}

Source : Les auteurs à partir des données PERVAL

Ces évolutions ne sont pas cantonnées au seul segment des appartements anciens puisque le segment des maisons anciennes connaît des évolutions similaires; le test ANOVA étant de nouveau très significatif ${ }^{28}$. Le montant moyen des transactions dans l'agglomération rémoise a progressé de 93,1 \% entre 1999 et 2006. Cependant, le marché est caractérisé par deux périodes différentes. Jusqu'en 2003, les prix ont tendance à croître à un rythme " modéré ". À partir de 2003, le montant moyen des transactions passe d'un indice 130,6 à 193,1 soit une progression de 47,9 \%. Là encore, les quartiers Clairmarais et Faubourg de Laon se distinguent puisque les prix moyens des maisons anciennes y progressent de 79,6 \% et 59,7 \%, soit les plus fortes croissances observées à Reims entre 2003 et $2006^{29}$ (cf. tableau 6).

Ces observations peuvent être le signe d'un certain intérêt pour un quartier qui accueille la gare assurant la liaison TGV directe vers Paris. Par ailleurs, la comparaison des prix par quartier par rapport aux prix moyens observés à Reims montre un rapprochement des prix immobiliers dans les quartiers Clairmarais et Laon et une croissance plus forte des prix à partir de 2004. Mais dans la mesure où d'autres quartiers contigus au centre-ville connaissent cette même évolution, il est difficile d'imputer clairement ces augmentations à la mise en service du TGV.

On peut, toutefois, noter que les quartiers proches de la gare bénéficient de la modification d'image et de l'urbanisme liée à la mise en service du TGV. Le quartier Clairmarais, encore récemment quartier d'arrière-gare, poursuit sa lente 


\section{Figure 2 - La localisation des quartiers Clairmarais et Faubourg de Laon par rapport à la gare TGV de Reims}

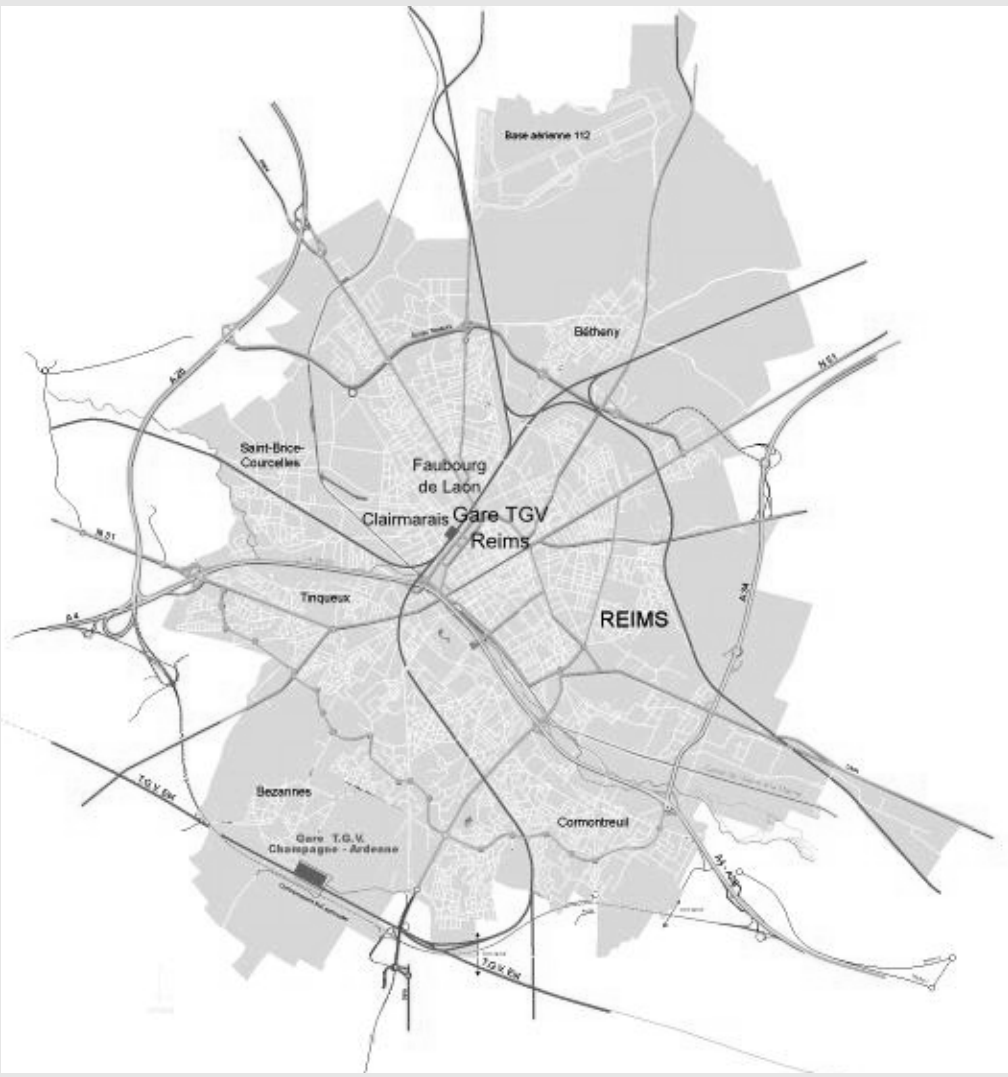

mutation. Outre près de $70000 \mathrm{~m}^{2}$ de bureaux qui seront, à terme, disponibles immédiatement derrière la gare, l'habitat résidentiel connaît des évolutions notables qui sont capitalisées dans les prix immobiliers, ce qui peut expliquer en partie leur progression localisée. Les pouvoirs publics ont mené une politique active visant à reconstruire le quartier de la gare. Ils ont favorisé la construction d'un quartier d'affaires immédiatement derrière la gare TGV tout en imposant l'existence de logements dans le quartier afin d'assurer une mixité de ses fonctions.

La progression des prix peut, enfin, résulter de phénomènes de spéculation. L'analyse de l'évolution de la rotation des logements et de la part des logements loués peut fournir des indications à ce sujet.

\subsubsection{Une accélération de la rotation des logements et une part croissante du nombre de logements occupés dans les transactions}

L'analyse de la date de la précédente transaction des biens immobiliers est riche d'enseignements. En effet, une accélération de la rotation des logements peut résulter 


\section{Tableau 6 - Évolution des prix des maisons anciennes de 1999 à 2006 dans les quartiers de Reims (base 100 en 1999)}

\begin{tabular}{|c|c|c|c|c|c|c|c|c|c|}
\hline Quartier & 1999 & 2000 & 2001 & 2002 & 2003 & 2004 & 2005 & 2006 & $\begin{array}{l}\text { Progres- } \\
\text { sion } \\
2006-2003\end{array}$ \\
\hline Centre & 100 & 91,5 & 135,2 & 90,7 & 109,8 & 151,0 & 149,6 & 167,3 & $52,4 \%$ \\
\hline Cernay & 100 & 113,5 & 133,9 & 127,0 & 135,5 & 134,8 & 168,8 & 198,6 & $46,6 \%$ \\
\hline Clairmarais & 100 & 102,7 & 114,6 & 106,9 & 93,4 & 114,1 & 179,4 & 167,7 & $79,6 \%$ \\
\hline Courlancy & 100 & 82,6 & 98,5 & 99,5 & 101,6 & 123,5 & 130,0 & 152,0 & $49,6 \%$ \\
\hline $\begin{array}{l}\text { Croix- } \\
\text { Rouge }\end{array}$ & 100 & 105,5 & 120,5 & 115,0 & 129,2 & 130,7 & 148,7 & 169,9 & $31,5 \%$ \\
\hline Jean-Jaurès & 100 & 102,1 & 109,3 & 109,4 & 164,3 & 121,1 & 162,4 & 157,2 & $-4,3 \%$ \\
\hline $\begin{array}{l}\text { La } \\
\text { Neuvillette }\end{array}$ & 100 & 115,7 & 117,4 & 135,0 & 138,0 & 151,8 & 162,4 & 175,0 & $26,8 \%$ \\
\hline Laon & 100 & 118,1 & 116,8 & 117,7 & 128,1 & 146,8 & 176,6 & 204,6 & $59,7 \%$ \\
\hline Moissons & 100 & 162,1 & 107,8 & 148,1 & 276,2 & 236,0 & 218,5 & 151,1 & $-45,3 \%$ \\
\hline Nord-Est & 100 & 98,7 & 97,0 & 114,0 & 137,1 & 170,7 & 126,0 & 182,2 & $32,9 \%$ \\
\hline Saint-André & 100 & 86,1 & 147,3 & 141,0 & 151,7 & 121,0 & 192,2 & 200,7 & $32,3 \%$ \\
\hline Saint-Anne & 100 & 94,5 & 113,1 & 121,1 & 150,3 & 141,9 & 181,7 & 227,0 & $51,0 \%$ \\
\hline Saint-Rémi & 100 & * & 58,7 & 69,7 & 87,6 & 140,7 & 105,3 & 229,0 & $161,4 \%$ ** \\
\hline Total & 100 & 110,4 & 118,7 & 123,0 & 130,6 & 148,0 & 166,6 & 193,1 & $47,9 \%$ \\
\hline
\end{tabular}

de deux phénomènes non exclusifs l'un de l'autre. D'une part, un changement rapide de propriétaires peut signifier qu'il existe des phénomènes de spéculation. Ainsi, la bulle spéculative du début des années 1990 s'explique, en partie, par le rôle joué sur les marchés immobiliers par les marchands de biens et les banques qui achetaient puis revendaient dans un laps de temps très court en engrangeant de substantielles plus-values lors de chaque transaction (RENARD, 2003). D'autre part, cette rotation rapide de biens immobiliers peut correspondre aux conséquences d'un quartier en pleine rénovation au sein duquel les biens sont achetés pour être rénovés et revendus ensuite. Cette dernière explication est un élément important dans la compréhension de l'évolution des prix dans les quartiers d'arrière-gare qui sont souvent rénovés du fait de la desserte ferroviaire à grande vitesse. Dans ce cas, il s'agit d'anticipations rationnelles liées à l'amélioration de l'attractivité future du quartier en raison d'une rénovation urbaine à venir.

Ainsi dans le quartier Clairmarais, le taux de rotation de maisons anciennes est globalement plus important que ce qui est observé en moyenne à Reims (cf. figure 3$)^{30}$ avec une accélération dans les années 2003 et 2004, années caractérisées par une augmentation rapide des prix dans ce quartier. Le changement rapide de propriétaires conduit à de fortes augmentations des prix.

Par ailleurs, l'apparition d'un nombre plus important d'investisseurs franciliens peut également résulter d'une nouvelle attractivité du marché immobilier rémois 


\section{Figure 3 - Part des transactions ayant muté la même année ou l'année précédente (marché de la maison ancienne)}

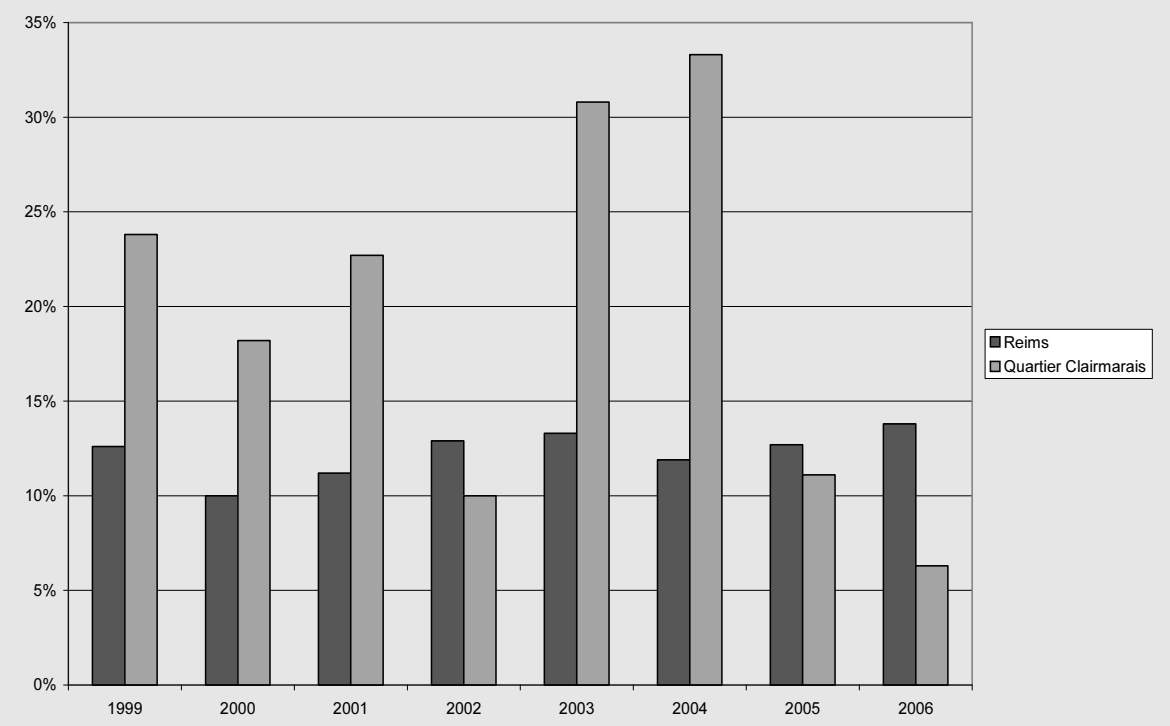

Source : Les auteurs à partir des données PERVAL

du fait du caractère « raisonnable » des prix observés à Reims par rapport à l'Ile-deFrance et de l'anticipation des conséquences futures de la LGV Est-européenne sur les prix immobiliers (cf. 3.2.2). Cet intérêt pour une acquisition immobilière dans l'agglomération rémoise peut correspondre à un investissement locatif visant ou non à bénéficier des dispositifs fiscaux en vigueur. Ceci est d'autant plus plausible que les acheteurs parisiens ont un profil socioprofessionnel différent de celui de l'ensemble des acquéreurs d'appartements neufs ou anciens (cf. tableau 7 pour le marché des appartements anciens). En effet, alors que les cadres, les professions intermédiaires et les employés représentent respectivement 25,2\%, 33,8 \% et 15,6\% de l'ensemble des transactions de ce segment en 2006, si l'on restreint l'analyse aux acheteurs parisiens, les cadres sont surreprésentés (55,9\%) au détriment des professions intermédiaires $(11,8 \%)$. Cette dépendance entre les PCS et l'origine géographique des acheteurs sur le marché des appartements anciens en 2006 est attestée par le test du Khi-deux ${ }^{31}$.

D'autres indicateurs montrent que les marchés immobiliers rémois intéressent les investisseurs. La croissance du nombre de logements occupés dans les transactions peut également laisser présager que des anticipations haussières sont à l'œuvre.

Longtemps, la part des appartements occupés dans les transactions immobilières était marginale. Alors qu'ils représentaient $1,4 \%$ des transactions sur ce segment en 1999, leur part passe à 9,5\% en 2004, à 13,2\% en 2005 et à 9,2 \% en 2006 (cf. tableau 8). À partir de 2004, ils représentent près d'une transaction sur dix. 
Tableau 7 - La Profession et Catégorie Sociale des acquéreurs d'appartements anciens en $\mathbf{2 0 0 6}$ dans l'agglomération de Reims

\begin{tabular}{|l|c|c|}
\hline \multirow{2}{*}{ PCS } & \multicolumn{2}{|c|}{ Appartements anciens } \\
\cline { 2 - 3 } & Toutes origines & Franciliens \\
\hline Agriculteurs & $2,6 \%$ & $0,0 \%$ \\
\hline Artisans, commerçants, etc. & $5,9 \%$ & $5,9 \%$ \\
\hline Cadres et prof. Intellect. sup. & $25,2 \%$ & $55,9 \%$ \\
\hline Employés & $15,6 \%$ & $17,6 \%$ \\
\hline Ouvriers & $8,5 \%$ & $0,0 \%$ \\
\hline Professions intermédiaires & $33,8 \%$ & $11,8 \%$ \\
\hline Retraités et inactifs & $8,3 \%$ & $8,8 \%$ \\
\hline Total & $100,0 \%$ & $100,0 \%$ \\
\hline
\end{tabular}

Source : Les auteurs à partir des données PERVAL

\section{Tableau 8 - La part des appartements anciens libres et occupés lors de l'achat de biens immobiliers dans l'agglomération de Reims de 1999 à 2006}

\begin{tabular}{|l|c|c|c|}
\hline Année & $\begin{array}{c}\text { Appartements } \\
\text { libres }\end{array}$ & $\begin{array}{c}\text { Appartements } \\
\text { occupés }\end{array}$ & Total \\
\hline 1999 & $98,6 \%$ & $1,4 \%$ & $100,0 \%$ \\
\hline 2000 & $98,9 \%$ & $1,1 \%$ & $100,0 \%$ \\
\hline 2001 & $99,7 \%$ & $0,3 \%$ & $100,0 \%$ \\
\hline 2002 & $99,1 \%$ & $0,9 \%$ & $100,0 \%$ \\
\hline 2003 & $97,5 \%$ & $2,5 \%$ & $100,0 \%$ \\
\hline 2004 & $90,5 \%$ & $9,5 \%$ & $100,0 \%$ \\
\hline 2005 & $86,8 \%$ & $13,2 \%$ & $100,0 \%$ \\
\hline 2006 & $90,8 \%$ & $9,2 \%$ & $100,0 \%$ \\
\hline
\end{tabular}

Source : Les auteurs à partir des données PERVAL

Plusieurs éléments expliquent cette évolution.

L'achat d'un bien occupé permet de bénéficier d'une décote à l'achat ce qui apparaît important lorsque les prix ne cessent d'augmenter. Par ailleurs, la progression des marchés, malgré la décote, permet au propriétaire de ne pas attendre la fin du bail pour le vendre tout en bénéficiant d'une plus-value substantielle. Cela permet à l'investisseur d'avoir immédiatement un locataire acquittant régulièrement ses loyers et ses charges. En outre, de plus en plus de ménages âgés envisagent de quitter leurs maisons individuelles pour un appartement. L'achat d'un logement occupé permet donc de choisir le bien adéquat dont ils pourront bénéficier plus tard à la fin du bail.

Dans le cas rémois, cela peut également être un signe d'anticipation des effets du TGV. Certains investisseurs prévoyant une augmentation importante des prix à l'arrivée du TGV acceptent de payer des prix élevés pour des biens loués. Cette acceptation conduit à réduire fortement la décote voire à la rendre négative sur certaines années (cf. tableau 9). 


\section{Tableau 9 - Le prix moyen $\mathbf{d u} \mathrm{m}^{2}$ des appartements anciens libres et occupés lors de l'achat de biens immobiliers dans l'agglomération de Reims de 1999 à 2006}

\begin{tabular}{|l|c|c|c|}
\hline Année & $\begin{array}{c}\text { Appartements } \\
\text { libres }\end{array}$ & $\begin{array}{c}\text { Appartements } \\
\text { occupés }\end{array}$ & $\begin{array}{c}\text { Décote } \\
(\%)\end{array}$ \\
\hline 1999 & 1076,85 & 775,86 & $28,0 \%^{*}$ \\
\hline 2000 & 1162,47 & 1231,00 & $-5,9 \%^{*}$ \\
\hline 2001 & 1177,15 & 361,06 & $69,3 \%^{*}$ \\
\hline 2002 & 1253,01 & 1320,29 & $-5,4 \%{ }^{*}$ \\
\hline 2003 & 1352,76 & 1147,18 & $15,2 \%$ \\
\hline 2004 & 1587,28 & 1535,58 & $3,3 \%$ \\
\hline 2005 & 1866,59 & 1639,73 & $12,2 \%$ \\
\hline 2006 & 2016,23 & 1990,72 & $1,3 \%$ \\
\hline
\end{tabular}

* peu significatif du fait du nombre restreint de transactions Source : Les auteurs à partir des données PERVAL

\subsubsection{L'apparition de nouveaux acteurs de la promotion immobilière qui anticipent une hausse des prix}

Le développement de l'offre immobilière s'accompagne également de l'arrivée sur le marché de nouveaux promoteurs. Alors que les promoteurs privés étaient longtemps cantonnés à de petites opérations compte tenu de l'omniprésence des bailleurs sociaux, depuis 2005, des promoteurs nationaux ou régionaux comme BouYGUES Immobilier, le groupe VINCI ou le promoteur lillois PALM ont acquis des emplacements prestigieux et s'engagent dans la réalisation d'opérations considérées comme étant haut de gamme. Il en va de même pour certains acteurs locaux qui se regroupent pour proposer, à court terme, des réalisations de ce type.

Plusieurs raisons peuvent être avancées. Premièrement, l'augmentation des prix conduit, en général, certains propriétaires à mettre en vente du foncier ou des immeubles "vétustes " afin de bénéficier de la phase croissante du cycle et de réaliser de substantielles plus-values ${ }^{32}$. La croissance des prix immobiliers aide à " la reconstruction de la ville sur elle-même " puisque cela permet à des biens supplémentaires d'être mis sur le marché et recyclés.

Deuxièmement, la progression des prix immobiliers observée à court terme sur certains segments dans les agglomérations ayant accueilli le TGV laisse augurer aux acteurs des prix de vente (des prix de sortie) en forte hausse rendant possibles certaines opérations préalablement non rentables ou permettant d'améliorer la rentabilité d'opérations déjà lancées.

La construction de logements a ainsi repris sur le territoire de Reims Métropole depuis 2003-2004 et s'est accompagnée d'une augmentation notable des prix à partir de 2004. Les prix des appartements qui ont été livrés en 2006, 2007 ou 2008 sont dès 2005 en forte augmentation ${ }^{33}$. 
Cette augmentation des prix peut résulter d'anticipations consécutives à la mise en place prévue du TGV. Les promoteurs anticipent que les acheteurs seront prêts à payer des logements neufs plus chers encore suite à la mise en service de la LGV participant ainsi à l'entretien de la croyance sociale selon laquelle les prix croissent au moment de la mise en service de la LGV.

Les promoteurs alignent leurs nouveaux projets sur le haut de gamme entraînant par là même une augmentation plus importante encore des prix des appartements neufs. Cette croissance rapide des prix des biens immobiliers neufs peut se propager à l'immobilier ancien par un report de la demande du segment du neuf vers l'ancien.

\section{-4 - Conclusion}

Dans les esprits, la mise en service d'une Ligne à Grande Vitesse est souvent associée à une flambée des marchés immobiliers résidentiels. Les marchés immobiliers rémois n'échappent pas à ce type d'anticipations. En effet, depuis l'annonce de la création de la LGV Est-européenne en 2000, de nombreux acteurs ont considéré que l'évolution des prix était imputable à ce projet et que cette tendance n'irait qu'en s'accentuant. Toutefois, l'analyse des transactions immobilières dans l'agglomération de Reims de 1999 à 2006 permet de relativiser les effets en amont de la mise en service de cette infrastructure sur la progression des prix immobiliers.

Force est de constater que de 1999 à 2006, les prix immobiliers ont progressé moins rapidement à Reims que dans les autres agglomérations de plus de 10000 habitants. Reims tenait le rang de 4ème ville la plus chère en 1999, elle apparaît au 10ème rang en 2005. Par ailleurs, l'augmentation des prix résulte, notamment, d'une augmentation de la demande locale liée à la progression du nombre de ménages rémois. Les franciliens demeurent peu présents sur ces marchés même s'ils sont plus nombreux dans certains quartiers.

Cependant, une desserte TGV conduit surtout à une augmentation des prix immobiliers localisée à proximité de la gare TGV. Des modifications des aménités et de l'urbanisme des quartiers proches de la gare liées aux politiques d'accueil et de valorisation du TGV menées par les collectivités locales et à un changement d'image des villes desservies y sont capitalisées dans les prix immobiliers. Le quartier d'arrière-gare connaît des progressions de prix plus importantes que la moyenne. Il est également caractérisé par une accélération des taux de rotation, notamment en 2003 et 2004.

Enfin, le TGV a tendance à redynamiser le secteur de la promotion immobilière porté par des acteurs locaux et nationaux.

Ce dynamisme est ainsi le signe d'anticipations partagées par de nombreux acteurs concernant l'influence de la mise en service d'une LGV sur les prix immobiliers, en général, dans les agglomérations desservies, alors que cette augmentation est observée principalement sur certains segments très localisés du marché. 


\section{Bibliographie}

ALONSO W., 1964, Location and Land Use, Harvard University Press, Cambridge.

Armstrong R.J., Rodriguez D.A., 2006, "An Evaluation of the Accessibility of Commuter Rail in Eastern Massachusetts using Spatial Hedonic Price Functions", Transportation, vol. 33, pp. 21-43.

AGENCE D'URBANISME ET DE DÉVELOPPEMENT DE LA RÉGION DE REIMS, 2005, Le marché immobilier rémois : prix actuels et tendances, Observatoire de I'Habitat de I'Agglomération Rémoise.

BAJIC V., 1983, "The Effect of a New Subway Line on Housing Prices in Metropolitan Toronto", Urban Studies, vol. 20, pp. 147-158.

BaZin S., BeCKerich C., De laplaCe M., 2006, Analyse prospective des impacts de la Ligne Grande Vitesse Est-Européenne dans l'agglomération rémoise et en région Champagne-Ardenne, Rapport final pour le Conseil Régional Champagne-Ardenne, 495 pages + annexes.

Bazin S., Beckerich C., Delaplace M., Masson S., 2006b, « La LGV-Est Européenne en ChampagneArdenne : quels effets sur la cohésion territoriale champardennaise », Revue d'Economie Régionale et Urbaine, $\mathrm{n}^{\circ} 2$, pp.245-261.

BAZIN S., BECKERICH C., DelAPLACE M., à paraître, "Hight Speed Railway, Service Innovations and Urban and Business Tourisms Development" in SARMENTO M.\& MATIAS A., Tourism Economics and Management : The State of the Art, Springer Verlag

BECKERICH C., 2001, Biens publics et Valeurs immobilières, ADEF.

Bessonne A.J., Heitz B., Boissinot J., 2005, "Marché immobilier : voit-on une bulle? ", Note de conjoncture, mars, INSEE.

BOWES D.R., IHLANFELDT K.R., 2001, "Identifying the Impacts of Rail Transit Stations on Residential Property Values", Journal of Urban Economics, ${ }^{\circ}$ 50, pp. 1-25.

CHAMBRE DES NOTAIRES DE LA MARNE, 2004, Le marché immobilier de la Marne.

CHAMBRE DES NOTAIRES DE REIMS, 2007, Données PERVAL, site internet.

CHAMBRE DES NOTAIRES PARIS ILE-DE FRANCE, 2007, Communiqué de presse du mois de juin.

CHAU K.W., NG F.F., 1998, "The Effects of Improvement in Public Transportation Capacity on Residential Price Gradient in Hong-Kong", Journal of Property Valuation and Investment, ${ }^{\circ}$ 16, pp. 397-410.

Chevalier J., 1995, Le Mans, 6 ans après I'arrivée du TGV, volumes I et II, Groupe de Recherche en Géographie Sociale, ESO, Espaces géographiques et sociétés, Université du Maine, Le Mans.

COMBY J., 1993, "Les six marchés fonciers : leur articulation avec les marchés immobiliers », Articulation du foncier et de l'immobilier, ADEF.

CORNUEL D., 1999, "L'hypothèse de bulle immobilière », Revue de l'OFCE, Observations de diagnostics économiques, $\mathrm{n}^{\circ} 70$, pp. 155-191.

DeYMIER G., 2005, Capitalisation immobilière des gains d'accessibilité : étude de cas sur l'agglomération lyonnaise, Thèse en sciences économiques, Université Lyon 2.

FujITA M., 1989, Urban Economic Theory, Land Use and City Size, Cambridge University Press.

IAURIF, INSEE, 2003, Atlas des franciliens, tome IV, Activité et Emploi.

INSEE, 2006, "Le SCOT de la région rémoise », Insee Flash Champagne-Ardenne ${ }^{\circ}$ 71, Insee ChampagneArdenne.

INSEE CHAMPAGNE-ARDENNE, 2006, Bilan économique 2005 - Bâtiment : toujours bien orienté en 2005, Dossier INSEE, $\mathrm{n}^{\circ} 20$.

INSEE, 2007, Chiffres clés, Résultats des enquêtes annuelles de recensement, Commune de Reims, site internet INSEE, juin

INSEE, 2008, Enquêtes annuelles de recensement de 2004 à 2007, site internet INSEE

INSEE, CHAMBRE SUPÉRIEURE DU NOTARIAT, 2007, Indice trimestriel des prix des logements anciens Province, site Internet INSEE.

MCDOnald J.-F., Osuı C.I., 1995, "The Effect of an Anticiped Transportation Improvement on Residential Land Values", Regional Science and Urban Economics, vol. 25, pp. 261-278. 
MTETMP/SESP, 2006, " Hausse générale de la construction neuve de logements, détente du marché dans les villes moyennes ", SESP en Bref, $n^{\circ}$ 9, Ministère des Transports, de l'Equipement, du Tourisme et de la Mer, Service Economie Statistiques et Prospective.

MuTH R.F., 1969, Cities and Housing, University of Chicago Press.

NAPPI-ChOulet I., 1994, Le marché des bureaux : analyse des facteurs de l'offre, Thèse pour le doctorat ès Sciences Économiques, Université de Paris XII.

OFFNER J.M., 1993, "Les effets structurants du transport : mythe politique, mystification scientifique », I'Espace géographique, $\mathrm{n}^{\circ} 3$, pp. 233-242.

ORLEAN A., 2002, Le rôle des croyances sociales en Economie, version pour I'atelier Economie Cognitive des 21 et 22 octobre 2002.

PAPAGEORGIOU Y.Y., 1973, "The Impact of the Environment upon the Distribution of Population and Land Values", Economic Geography, n 49, pp. 251-256.

PAPAGeOrGiou Y.Y., 1990, The Isolated City State, Routledge, London - New York.

RENARD V., 2003, Quelques caractéristiques des marchés fonciers et immobiliers, document de travail.

RICS, Royal Institution of Chartered Surveyors, 2002, Land Value and Public Transport, RICS Policy Unit, London.

RUBINSTEIN M., 2008, "Le marché de l'immobilier résidentiel en France : évolutions récentes et perspectives ", Revue d'économie financière $\mathrm{n}^{\circ} 91$.

SEGEFA, Service d'Etude en Géographie Économique et Fondamentale et Appliquée, 2001, Les choix résidentiels des ménages face à la problématique du développement durable, Université de Louvain.

YIU C.Y., WONG S.K., 2005, "The Effects of Expected Transport Improvements on Housing Prices", Urban Studies, vol. 42, $\mathrm{n}^{\circ} 1$, pp. 113-125.

WINGO L., 1961, Transportation and Urban Land - Resources for the Future, John Hopkins, Baltimore.

\section{Notes}

1 - Bezannes qui est un petit village séparé actuellement de l'agglomération rémoise par 500 mètres de terres agricoles, fait partie de Reims Métropole, la Communauté de Communes de Reims.

2 - Depuis le 14 décembre 2008 cependant, un aller-retour vers le Luxembourg et un allerretour supplémentaire vers Paris ont été mis en service.

3 - Nous limiterons notre analyse aux conséquences de la desserte TGV de la gare de Reims qui assure la quasi-totalité de la desserte de Paris.

4 - Les calculs présentés ont été effectués à partir d'une base de données représentant $47 \%$ des transactions immobilières par année de 1999 à 2006 (tirage aléatoire).

5 - Pour une analyse des déterminants des évolutions récentes du marché immobilier résidentiel français, cf. M. RUBINSTEIN, 2008.

6 - D'autres facteurs expliquent évidemment la mobilité résidentielle des ménages. Ainsi, l'inadéquation du logement, les évolutions de la composition du ménage, l'activité professionnelle et la nature du voisinage sont autant de raisons de déménagement (SEGEFA, 2001).

7 - En tous cas pour les villes desservies comparables à Reims en termes de taille et de distance à Paris (Tours, Le Mans par exemple) et ce, comparativement aux autres villes (BAZIN et al., 2006a).

8 - Si pour des raisons d'aménagement du territoire, des rames TGV empruntent le réseau ferroviaire classique puis le réseau à grande vitesse pour rejoindre Paris au départ d'agglomérations plus petites, les dessertes très limitées ne peuvent être comparées à une desserte autoroutière. 
9 - Comme beaucoup de villes desservies par le TGV, Reims n'a pas échappé pas à cette logique de restructuration et de recherche de changement d'image préalable à la mise en service du TGV (cf. II).

10 - Ces croyances sociales prennent la forme suivante : "l'individu i croit que le groupe croit que la proposition P est vraie " (ORLEAN, 2002). Elles remettent en question le caractère rationnel du comportement individuel des agents.

11 - Cf. les nombreux programmes de construction de locaux à proximité des gares TGV (Novaxis au Mans, Euralille à Lille, etc.)

12 - Par exemple, l'examen des permis de construire dans les communes de la CUM hors du Mans montre que le nombre moyen de permis de construire déposés par an est passé de 90 de 1983 à 1988 avant l'arrivée du TGV à 177 de 1989 à 1993 (CHEVALIER, 1995).

13 - En effet, des liens existent entre les marchés immobiliers et fonciers. Même si le sens de la liaison fait toujours débat, l'évolution des marchés immobiliers conditionne l'orientation des marchés fonciers à moyen terme (COMBY, 1993). La valeur du bien final qui s'échange sur le marché immobilier va déterminer la valeur du foncier par l'intermédiaire du compte à rebours du promoteur. L'augmentation des prix immobiliers peut conduire les promoteurs à offrir des prix plus élevés pour obtenir des terrains. Cette augmentation de la charge foncière peut convaincre des propriétaires de céder leurs terrains aux promoteurs. Le nombre de transactions sur le foncier augmente donc lors des périodes de croissance des prix immobiliers et conduit à terme à un nouveau dynamisme de la promotion immobilière.

14 - INSEE - Recensement Général de la population, 1999.

15 - Pour une analyse de la littérature relative à l'évolution récente du marché immobilier résidentiel en France, cf. RUBINSTEIN, 2008.

16 - Chaque acquéreur devait débourser 2015 euros par $\mathrm{m}^{2}$ pour un appartement ancien et 2764 euros par $\mathrm{m}^{2}$ pour les appartements neufs en 2006.

17 - L'existence d'une éventuelle corrélation entre l'importance des bailleurs sociaux et le niveau élevé des prix dans l'agglomération est en débat.

18 - On peut toutefois observer que le nombre de transactions diminue sur le segment des appartements neufs en fin de période, il peut s'agir d'une stratégie des promoteurs pour proposer des programmes au moment de la mise en service de la LGV afin de bénéficier pleinement de l'augmentation des prix qu'ils anticipent (cf. 2.3.3.) Par ailleurs, l'évolution du nombre de transactions doit être utilisée avec prudence puisque les évolutions peuvent en partie résulter d'une participation plus ou moins grande des notaires d'une année à l'autre au renseignement de la base.

19 - Ces données comprennent la construction HLM. Depuis 2000, on assiste à un rééquilibrage entre les appartements autorisés à la construction pour une location future qui tendent à diminuer et les logements destinés à la vente dont le nombre progresse (AUDRR, 2005).

20 - La première raison est la faiblesse des réserves foncières constituées par la collectivité publique. La seconde raison qui peut apparaître comme étant paradoxale est la domination sur le marché des trois organismes HLM de la ville (OPAC, Foyer Rémois et L'Effort Rémois) qui ont constitué des réserves foncières importantes qu'ils gèrent au mieux de leurs intérêts, et qui laissent ainsi très peu de place aux constructeurs du secteur libre. La troisième raison est liée à la nature de l'utilisation des terres à la périphérie de Reims. D'une part, la zone de l'AOC Champagne est en effet à proximité de l'agglomération et les prix à l'hectare de la vigne n'incitent évidemment pas à un changement d'usage de ces terres. D'autre part, la Champagne-Ardenne est caractérisée par une agriculture dynamique dont les dirigeants préservent l'utilisation agricole des terres à l'aide des SAFER.

21 - Analyse des fichiers SITADEL disponibles sur l'agglomération de Reims. 
22 - Le taux de croissance annuel moyen de la population passe de 0,66 \% sur la période 1975/1990 à 0,41 \% de 1990 à 1999.

23 - Le Schéma de cohérence territoriale (SCOT) est un document de planification stratégique destiné à mettre en cohérence les politiques menées par les différentes collectivités d'un territoire. Il vise ainsi à définir un véritable projet de développement de ce territoire.

24 - Cf. RUBINSTEIN, 2008.

25 - La comparaison des prix immobiliers montre qu'à Reims les prix sont bien plus faibles qu'à Paris ou dans les communes de l'Est parisien aussi bien sur le segment des appartements anciens que des maisons anciennes. En 2006, les prix moyens des appartements anciens au $\mathrm{m}^{2}$ sont de $5772 €$ à Paris intramuros avec des prix les plus faibles dans les arrondissements de l'Est de Paris à savoir entre $4571 €$ dans le 19 e arrondissement et $5358 €$ dans le 11 e arrondissement. Les prix pratiqués dans les communes de l'Est de Paris sont plus faibles : moins de $2500 €$ pour les moins recherchées comme Tremblay-en-France, Bobigny ou Clichy-sous-Bois, et entre 2500 et $3000 €$ pour des villes de la seconde couronne comme Chelles ou Torcy et avec des prix plus élevés encore pour les communes de la première couronne. En 2006, le prix des appartements au $\mathrm{m}^{2}$ n'était que de $2016 €$ à Reims. En ce qui concerne le prix des maisons anciennes, on retrouve cette différence entre prix rémois et ceux de l'Est parisien. A Reims, le prix médian est de $200000 €$ contre $256000 €$ à Torcy, $275000 €$ à Chelles et $300000 €$ à Montreuil.

26 - Les montants des mensualités d'un prêt pour l'achat d'une maison au prix médian observé à Reims, Torcy ou Montreuil sont respectivement de $1181 €, 1493 €$ et $1771 €$ (hors assurance et à un taux de 3,70\% sur 20 ans, taux moyen observé en 2006). A ces mensualités, il convient d'ajouter les coûts de transports soit 450 euros mensuels hors frais de réservation pour les déplacements domicile-travail en TGV (prix classique de l'abonnement) pour une localisation à Reims et 112 euros mensuels maximum pour une localisation en région parisienne (carte orange). Les avantages d'une localisation sont alors réduits voire inexistants. Par ailleurs, les coûts de transports représentent une dépense alors que des mensualités plus élevées destinées à acquérir un bien immobilier contribuent à accroître son patrimoine.

27 - L'influence du quartier sur le prix par $\mathrm{m}^{2}$ des biens immobiliers est très significative. Pour le marché des appartements anciens, F est égal à 76,27 avec 1-p > 99,99\%.

28 - L'influence du quartier sur le prix des biens immobiliers est très significative. Pour le marché des maisons anciennes, F est égal à 540,48 avec 1-p >99,99\%.

29 - A l'exception d'un autre quartier qui connaît un nombre de transactions très faible.

30 - La diminution du taux de rotation les deux dernières années pourrait s'expliquer par l'attente de l'arrivée du TGV ou par la diminution du nombre de biens disponibles à rénover.

31 - Khi-2 = 19,76, ddl = 6, 1-p = 99,69\%. La dépendance est également marquée pour les années antérieures, mais la présence plus limitée des acheteurs franciliens réduit la significativité du test.

32 - C'est le cas notamment de Maisons de Champagne comme Piper-HeIDsieck qui a cédé ses locaux dans l'agglomération.

33 - L'analyse des ventes en l'état futur d'achèvement permet de mesurer dans une certaine mesure les anticipations des promoteurs quant au niveau des prix au moment de la livraison du logement. Les prix au $\mathrm{m}^{2}$ s'établissent respectivement à 2617, 3414 et 3621 euros selon la date de livraison à savoir en 2006, 2007 et 2008 (calculs réalisés par les auteurs à partir des données PERVAL). 